\title{
LOS DIPUTADOS DEL PARLAMENTO GALLEGO EN FACEBOOK
}

\author{
Rúas Araújo, José ${ }^{1}$ \\ Dapena González, Borja² \\ Universidad de Vigo \\ joseruas@uvigo.es \\ borjamultimedia@yahoo.es
}

Material original autorizado para su primera publicación en la revista académica REDMARKA. Revista Digital de Marketing Aplicado

https://doi.org/10.17979/redma.2011.01.07.4728

Recibido: 17 Octubre 2011

Aceptado: 1 Diciembre 2011

\section{Resumen}

El objetivo de este estudio es analizar la presencia y utilización de la red social Facebook por parte de los diputados del Parlamento de Galicia, partiendo de los datos más recientes de audiencias y del repaso de diferentes investigaciones sobre la actividad de los parlamentarios españoles en la red (mails, páginas web, blogs, Facebook y Twitter), y comparando los resultados obtenidos en cada caso.

Se trata de una investigación cuantitativa, de carácter exploratorio y longitudinal, a través del rastreo de las cuentas en Facebook de los representantes del Parlamento gallego a lo largo de cinco meses, y del seguimiento diario de la actividad en la red de los diputados, observando las distintas variables y posibilidades ofrecidas en el empleo de esta herramienta por parte de los 75 miembros de la Cámara autonómica que integran el universo de estudio.

En concreto, se observaron todas las actualizaciones de estado realizadas, comentarios, enlaces, fotografías y vídeos visibles en el muro de cada

\footnotetext{
${ }^{1}$ Profesor titular de "Comunicación Electoral e Institucional" de la Facultad de Ciencias Sociales y de la Comunicación de la Universidad de Vigo. Autor de: Manual del Candidato Electoral. Madrid: Catarata, 2011; Quero ser presidente. Claves da Comunicación Electoral. Santiago de Compostela: Alvarellos Editora, 2008.

${ }^{2}$ Doctorando de la Facultad de Ciencias Sociales y de la Comunicación de la Universidad de Vigo y Licenciado en Publicidad y Relaciones Públicas. Máster en Investigación en Comunicación.
} 
parlamentario, calculando sus medias y ranking de actividad, además de comprobar la lista de amigos, grupos y páginas agregadas o el idioma empleado. Para acceder al Facebook de los diputados gallegos se utilizó la técnica de la simulación, a través de la observación participante, además de la información institucional disponible en la red y los datos complementarios solicitados a los distintos grupos parlamentarios.

Los resultados muestran que la evolución en la utilización de Facebook es mayor en la sociedad que en los parlamentarios del Congreso de los Diputados y el Parlamento de Galicia y que, si bien en los representantes gallegos se observa mayor presencia que en los anteriores, la utilización de esta herramienta es muy similar en ambos casos, pues apenas la tercera parte de ellos registran cierta actividad, siendo prácticamente testimonial en la mayoría de los diputados estatales y autonómicos analizados.

Palabras clave: Parlamento/Redes Sociales/Facebook

\section{Abstract \\ MEMBERS OF GALICIAN PARLIAMENT IN FACEBOOK}

The aim of this study is to analyze the presence and use of social networks, particularly Facebook on behalf of Members of Galician Parliament delivering, the most recent data of audience and the different investigations about the Spanish Members of Parliament activity on the net (mails, blogs, Facebook, webs and Twitter) and comparing the results in each case.

For the past five months, a quantitative, exploratory and longitudinal tracking of the Facebook accounts which belong to the Members of the Galician Parliament has been carried out, also a daily follow-up of the updates of the state and the posting of comments, links, photos, videos and questions on the wall of 75 parliamentarians. 
To access the Facebook of each member, a simulation technique will be used, through participant observation, a task that will complement the description of the tools available through site and official links of the Galician Members of the Parliament and the requested information to the different parliamentary groups.

The results show that the evolution of the use of Facebook is greater in the society than in Members of the both the Spanish and Galician Parliament, and in the case of the Galician Parliament there is greater presence than in the former. Although the use of this tool is quite similar in both cases, it is almost testimonial in reference to most of the Members of the Galician or Spanish Parliaments.

Key words: Parliaments/Social Networks/Facebook 


\section{Introducción}

Los estudios de audiencias realizados en España muestran, durante la última década, el crecimiento exponencial registrado en Internet con respecto a otros medios. De acuerdo con los datos de la Asociación para la Investigación de Medios de Comunicación (AIMC), desde el año 2000 hasta el 2007, la audiencia de Internet registrada en el público de 14 o más años experimentó un incremento del $367,86 \%$, tal y como se indica en alguno de los trabajos publicados a este respecto (Larrañaga Rubio, 2008), en el que también se destaca que, en el año 2007, Internet ya resultaba una fuente fundamental de información para, prácticamente, la mitad $(49,8 \%)$ de los internautas españoles encuestados.

Asistimos, durante el último decenio, a la evolución en el consumo de Internet entre la población adolescente y, en consecuencia, entre el target de los primeros votantes y electores más jóvenes, acompañado del progresivo cambio en los hábitos de consumo y trasvase de audiencias desde el medio televisivo offline (fundamental en el ejercicio de la comunicación política durante la segunda mitad del siglo XX), hacia Internet y el consumo televisivo online.

Si observamos los resultados de la última encuesta realizada por AIMC a usuarios de Internet $^{3}$, referida al año 2010, y la comparamos con la realizada un año antes ${ }^{4}$, comprobaremos un par de datos significativos.

Por un lado, que continúa la progresiva disminución -ya observada en ediciones anteriores del mismo estudio- del tiempo de consumo de los denominados medios tradicionales, con un $69,4 \%$ de personas que declaran haber reducido el tiempo de ver la televisión en favor de Internet.

\footnotetext{
${ }^{3}$ Estudio "Navegantes en la Red: 13 encuesta AIMC a usuarios de Internet" publicada en febrero de 2011, obtenida a través de una muestra probabilista y representativa de la población española basada en la realización de 30 mil entrevistas al año, en tres oleadas de 10 mil entrevistas cada una. Cfr. http://download.aimc.es/aimc/navred2010/macro2010.pdf [Recuperado 25-04-2011]. ${ }^{4} 12^{\mathrm{a}}$ encuesta Navegantes en la Red, también de AIMC. Disponible en: http://download.aimc.es/aimc/03internet/macro2009.pdf [Recuperado 25-04-2011].
} 
Pero por otra parte, también se constata, con respecto a la utilización de redes sociales, el menor crecimiento (o incluso estancamiento) en la utilización de blogs y páginas webs personales, frente al crecimiento en la utilización de Facebook.

El 73,6\% de los encuestados reconocía haber accedido a la utilización de redes sociales durante los días previos a la consulta (60,3\% el día anterior y el 13,3\% durante los últimos 7 días), frente al $58,6 \%$ de los mostrados en la encuesta realizada en 2010, que reconocían haber accedido durante los últimos días (43,5\% el día anterior a la realización de la encuesta y $15,1 \%$ en los últimos 7 días).

De ellos, casi el 90\% (89,9\%), declara haber utilizado la red Facebook durante el último año, seguida de Tuenti $(26,2 \%)$, Twitter $(21,9 \%)$ y Linkedln $(14,3 \%)$. Un resultado notablemente mayor en la utilización del Facebook con respecto a la que en la encuesta del 2010, cuyos datos mostraban el siguiente "registro" de ususarios de redes sociales: 61,7\% Facebook, 20,8\% Tuenti, 13\% Windows Live, $10,1 \%$ MySpace y $8,7 \%$ Twitter. Un crecimiento, por tanto, de casi el $30 \%$ en la utilización de Facebook registrado durante el último año con respecto al anterior, frente a otro más modesto (entre el 5,4 y el 5,6\%) correspondiente a herramientas como Tuenti y Twitter, respectivamente.

En cambio, se muestra un estancamiento en la utilización de páginas web personales entre las mediciones de los dos últimos años, con un $82 \%$ de usuarios que reconocen que no disponían de esta herramienta en el estudio del 2010, el mismo porcentaje que en 2011, y un leve crecimiento en cuanto a la disponibilidad y utilización de blogs. Concretamente, del $4,1 \%$ de usuarios $(71,9 \%$ frente al $67,8 \%)$ que declaraban disponer de blog durante el último año con respecto al anterior. $Y$ entre los que declaran disponer de blog en la última de las encuestas de AIMC, el 10,6\% reconoce que no lo actualiza, el $10 \%$ que lo actualiza ocasionalmente y tan sólo el $7,4 \%$ que lo hace frecuentemente.

\footnotetext{
${ }^{5}$ Cuyos resultados tan sólo se esbozaban a través de la pregunta de si "está usted registrado en alguna red social", lo cual no implicaba su utilización, un matiz en el que se ya se incide en la edición del 2011.
} 
Al comparar los resultados anteriores con algunos de los estudios realizados sobre la presencia y utilización de las redes sociales por parte de los parlamentarios españoles, se pueden observar algunas diferencias con respecto a la utilización de Internet y las redes sociales, entre los cibernautas y sus representantes políticos del Congreso de los Diputados.

Diferencias constatables en algunos de los estudios realizados a comienzos de la pasada década sobre el grado de interactividad existente entre ciudadanos y representantes del Congreso y el Senado entre 1999 y 2001 (Dader, 2001: 203), en los que ya se podía observar que el incremento, en casi un $30 \%$, de parlamentarios de ambas cámaras que hacían pública su dirección electrónica. Un dato que, sin embargo, no se correspondía con el porcentaje de parlamentarios que respondían a través de sus cuentas electrónicas a los requerimientos del experimento planteado en el citado estudio, ya que apenas superaban el $16 \%$ de respuestas.

Un hecho que podría explicarse, tal y como se indicaba en el mismo estudio, por una iniciativa institucional de equipamiento generalizado, más que por la voluntad expresa de sus señorías, así como por el impulso, durante esa legislatura, a la constitución de una comisión parlamentaria de fomento de las nuevas tecnologías.

Dader y Campos (2006:105), repitiendo la experiencia realizada años atrás, observaron que sólo un $17,4 \%$ de los diputados y senadores respondieron a las peticiones de información, mostrando un estancamiento justificable, en parte, por el incremento del tráfico de mensajes con respecto al período anterior, pero que, en cualquier caso, revelaba índices menores que los registrados en las cámaras de otros países europeos.

Partiendo de la observación de la propia página web del Congreso de los Diputados, algunos de los estudios realizados hasta ahora (Cotarelo, 2010) concluyen que, aunque la penetración de Internet es menor que la registrada en los partidos políticos (hecho que se atribuye en parte a la asunción de cierta disciplina colectiva del partido frente al riesgo que supone una exposición 
individual y directa de cada diputado), es sensiblemente igual a la registrada en el conjunto del ciberespacio, de acuerdo con el Estudio General de Medios (EGM). Sobre todo si se tiene en cuenta, según el mismo estudio, que son 89 los diputados (en realidad 87, pues el enlace de dos de ellos no está operativo), sobre el total de los 350 del Congreso, que disponen de blog o página web, lo cual significa, aproximadamente, un 25\%. De los 87 bitácoras en activo, se actualizaba diariamente un $5,7 \%$, un $44,8 \%$ al menos una vez por semana y el $36,7 \%$ de forma ocasional.

Sin embargo, si comparamos estos datos con los comentados anteriormente, alusivos a los últimos estudios de audiencias, se observan ciertas evoluciones y distanciamiento, tanto desde el punto de vista cuantitativo, como cualitativo, en cuanto a la falta de reciprocidad en las comunicaciones a través de la red entre ciudadanos y políticos y el escaso interés mostrado por los contenidos políticos. Variaciones, todavía mayores, en la utilización de las nuevas herramientas como Facebook, entre los parlamentarios españoles y la sociedad en general.

En las conclusiones del "Informe sobre política y redes sociales" elaborado por la agencia Intelligence Compass $^{6}$, se muestra que los ciudadanos buscan en las redes sociales relacionarse con su entorno más cercano, pero excluyen de éste a los políticos. $Y$ de los encuestados que afirmaron tener en su red social a políticos, era porque declaraban vincularse con ellos por amistad, pero no por motivos políticos (únicamente 49 de 722 personas reconocían que seguían a los políticos en su red por afición o interés), teniendo en cuenta además que un buen número de consultados afirmaban no incluir a políticos en sus redes sociales para que no les vinculasen con ningún partido.

El mismo estudio ponía de manifiesto la baja probabilidad de que el flujo de la "ola" de la utilización de las redes sociales de la campaña del presidente Barack Obama llegase a España en las elecciones municipales de mayo del 2011, debido al escaso interés de la ciudadanía por los contenidos políticos en la red.

\footnotetext{
${ }^{6}$ Basado en encuestas realizadas entre agosto y septiembre de 2010 a una muestra de 1.550 políticos y particulares en España, tratando de investigar las relaciones producidas entre ambos a través de la utilización de herramientas como Facebook, Twitter, Linkedln y Xing. Disponible en: www.intelligencecompass.com [Recuperado 27-04-2011].
} 
Desde instancias diversas se ha fomentado la presencia de la vida parlamentaria a través de la red. Las distintas cámaras, autonómicas y estatal, ofrecen incluso la posibilidad de acceder a debates televisados a través de sus webs oficiales y los parlamentos impulsan la formación de sus señorías en la utilización de las nuevas tecnologías, tanto de forma individual, como colectiva, a través de la publicación de normas para la creación de páginas y sitios web parlamentarios. Prueba de ello es el documento "Directrices para sitios web parlamentarios" difundido en marzo de 2009 por la Unión Interparlamentaria de Naciones Unidas a través del Centro Global para TIC en los Parlamentos en el que, entre otros aspectos, se ofrecen una serie de recomendaciones prácticas a la hora de planificar y supervisar los sitios web de los parlamentos, con normas concretas a los diseñadores, promotores y administradores de los mismos.

Pero la siguiente cuestión que se plantea es el logro en paso de una transparencia pasiva, por la que los ciudadanos pueden solicitar la información que consideren oportuna, estableciendo en las instituciones la obligación de responderles, a otra transparencia activa. Una tarea que va más allá de la simple transmisión unidireccional y centralizada de información, que busca adaptarse a los intereses de cada grupo ${ }^{7}$ e individuo y que está abierta al diálogo, superando el plano testimonial y propagandístico reducido a operadores políticos (Rubio, 2011: 26).

La cuestión, también, de si la supuesta reconversión tecnológica de la política va acompañada de una modificación de los comportamientos y actitudes por parte de los representantes políticos (Rúas, 2011: 132) y sobre si los problemas de funcionamiento democrático son de raíz cultural antes que tecnológica, de modo que Internet difícilmente podrá alterar, por sí sola, los hábitos políticos.

Porque incluso algunos de los estudiosos que observan el incremento de la capacidad de movilización de los partidos políticos a través del uso de herramientas de la política informacional y el importante papel desempeñado por Internet a la hora de facilitar una relación directa entre partidos, candidatos y

\footnotetext{
${ }^{77}$ Grupos de trabajo o interés a través, por ejemplo, de la creación colaborativa de contenidos (Wiki).
} 
votantes (Castells, 2009: 387), también ofrecen muestras sobre la decadencia de su utilización e instrumentalización por parte de quienes detentan el poder (Castells, 2001).

De ahí la necesidad de un cambio que se plantea sobre la asunción de la denominada "Actitud 2.0" (Fages-Ramió, 2010) que entiende la participación más allá de la simple presencia, pues exige también la contribución activa, a través de la búsqueda de la retroalimentación y el intercambio de opiniones y contenidos.

De hecho, técnicamente resulta pertinente la distinción, en cuanto a diferencia de intención, entre multipresencia (políticos y candidatos que utilizan y vinculan varias herramientas incrustadas a la vez, como página web, blog grupal y blog individual), presencia testimonial, y el propio concepto de conversación (Fernández Crespo, 2007:159), que va más allá de la simple acción de colgar sus post o dejar que los internautas escriban sus comentarios sin proceder luego a responderles.

Además, la discusión sobre política con amigos o compañeros no suele considerarse participación, si bien la irrupción de las redes sociales plantea algunas diferencias a la hora de cuestionarse si escribir comentarios políticos a través de estas nuevas herramientas es o no participación, teniendo en cuenta que los nuevos medios comienzan a ejercer su propio papel en la definición de la agenda mediática y, en consecuencia, política (Drezner y Farrell: 2008).

Algunos de los datos reflejados hasta ahora a través del CIS en sus estudios sobre internet y participación política en España (Anduiza et al.: 2010), muestran un incremento progresivo en el número de ciudadanos que se informan sobre asuntos políticos a través de Internet y, además, que los estímulos negativos, de circulación de mails criticando a políticos $(27 \%)$, son más frecuentes que los positivos (11\%). Impactos más o menos directos sobre la participación política, pero en cualquier caso difíciles de cuantificar, teniendo en cuenta la consideración de que los usos políticos de Internet o el simple consumo de información política no constituyen actividades participativas como tales. 
Atendiendo a la clasificación de Dahlgren (2005:153) sobre las distintas formas de interactuación política, redes sociales como Facebook y Twitter entrarían dentro de las denominadas como de carácter "parapolítico" pues, aunque no tratan propiamente sobre aspectos políticos, los contenidos que circulan a través de las mismas pueden llegar a tener, de forma implícita, elementos de tal carácter que deriven hacia cuestiones politizadas (Resina, 2010:146).

Sin embargo, más allá de las referencias indicadas hasta ahora en cuanto a la utilización del correo electrónico por parte de los políticos y la observación de la presencia de los parlamentarios a través de los accesos oficiales desde la web del Congreso de los Diputados y sus blogs, tampoco parece que el empleo de nuevas herramientas que ofrecen las redes sociales como Facebook, contribuya a mejorar esa comunicación entre los diputados españoles y sus representados.

En este sentido, alguno de los estudios realizados hasta la fecha sobre la comunicación de los parlamentarios españoles en Facebook (Túñez y Sixto: 2011) muestra que un tercio de los diputados con enlace propio desde la web del Congreso $(31,96 \%)$ referencia actividad en alguna red social, algo que, proyectado sobre el conjunto del hemiciclo, tan sólo significa el 8,58\% de los 350 miembros de esta Cámara. Una proporción todavía menor en el caso concreto de Facebook, donde tan sólo uno de cada cuatro parlamentarios estatales $(25,14 \%)$ disponen de perfil o página personal.

El mismo estudio refleja que 97 de los 350 diputados del Congreso disponen, oficialmente, a través de la página web del Congreso, de enlaces a páginas personales, la mayoría de ellos mediante blogs $(80,41 \%$, lo que representa un $22,28 \%$ sobre el total), pero sólo un $13,4 \%$ usan Facebook (un 3,71\% sobre el total de escaños), y el $12,37 \%$ Twitter (3,43\% sobre el total), una cifra que se reduce si se tiene en cuenta los diputados que tienen perfil abierto, es decir, a los que puede acceder cualquier usuario de la red (tan sólo el 16,28\% de los diputados dispone de perfil abierto).

Una presencia, además, mayoritariamente testimonial (el $48,87 \%$ de los diputados con página o perfil en Facebook no ofrece ninguna información y un $28,4 \%$ se 
limita a publicar datos básicos), con escasas aportaciones personales y pocas actualizaciones y comentarios publicados por los usuarios (7,53 de media) con respecto a las entradas realizadas por los propios diputados.

En el caso de Twitter, la observación de su ranking nos muestra, dentro del "top" de los 50 con más seguidores, únicamente dos instituciones políticas y gubernativas, como son el Europarlamento, que ocupa el puesto número $18 \mathrm{y}$, en el caso de España, el Twitter de La Moncloa que, seguido por un total de 128.829 personas, ocupa el puesto 33. Fútbol y música copan los primeros puestos en un Twitter cuyo ranking, en la categoría política, encabeza el lehendakari vasco Patxi López, con 105.499 seguidores, delante de partidos como el PSOE (seguido por 17.430 personas) y PP (14.322 seguidores) ${ }^{8}$.

Twitter tiene la ventaja de que la información que circula por esta red está estructurada y se puede medir cómo se propaga (RT) y cómo se dialoga (Reply). Esto ha permitido observar que la información fluye por comunidades de usuarios que poseían entre ellos unos vínculos más fuertes que con el resto de la red, como se describe en alguna de las muestras recogidas con motivo de las últimas elecciones catalanas (Congosto et al., 2011:13), donde se comprobó que los usuarios se agrupaban por afinidad política a la hora de transmitir y compartir información.

Precisamente, en un análisis de las cuentas Twitter del Parlament de Calalunya, se detectó, en el seguimiento realizado entre marzo y abril del 2010, la existencia de una audiencia de casi cinco millones de cibernautas, generada a través de los más de 16.000 usuarios de Twitter que seguían alguna o varias de las cuentas de sus diputados, a través del análisis de la etiqueta "parlament". catalán que, por aquel entonces, contaba con un total de 20 diputados activos en Twitter que utilizaban esta herramienta de forma regular, lo cual representa un $14,8 \%$ sobre el total de los 135 representantes que componen la cámara autonómica catalana.

\footnotetext{
${ }^{8}$ Cfr. http://twitter-espana.com/ranking/top-50/ [Recuperado 02-05-2011].

${ }^{9} \mathrm{Cfr} . \mathrm{http}: / /$ www.kgovernment.com/2010/04/19/anlisis cuentas twitter parlament de catalunya abril/ [Recuperado 02-05-2011].
}

REDMARKA UIMA-Universidad de A Coruña - CIECID 


\section{Objetivos y metodología}

Partiendo de la descripción general del empleo de internet y las redes sociales por parte de los representantes del Congreso de los Diputados, el objetivo específico de este estudio es observar la presencia y utilización de la herramienta Facebook por parte de los diputados de los tres partidos con representación en el Parlamento de Galicia (PPdeG, PSdeG-PSOE y BNG) y su comparación con respecto a los miembros de la Cámara Baja.

Se trata de una investigación cuantitativa, de carácter exploratorio y longitudinal, a través del rastreo de las cuentas en Facebook de los diputados gallegos a lo largo de cinco meses, en el período comprendido entre octubre de 2010 y febrero de 2011, y el seguimiento diario completo de todas las actualizaciones de estado, publicaciones de comentarios y enlaces, fotografías y vídeos en el muro de cada parlamentario, comprobando además la lista de amigos, grupos y páginas agregadas a su perfil ${ }^{10}$.

Para poder acceder a la información de cada diputado se utilizó la técnica de la simulación, a través de la observación participante, con la creación de una cuenta y perfil de usuario ficticios, tarea contrastada con la información solicitada a los distintos grupos parlamentarios.

\footnotetext{
${ }^{10}$ De acuerdo con Wikipedia, la enciclopedia libre más popular de la red, el término "muro" (wall en inglés), es el espacio en cada perfil de usuario que permite que los amigos puedan escribir y compartir sus mensajes. A través de la lista de amigos, el usuario puede agregar a cualquier persona que conozca y esté registrada, siempre que acepte su invitación. Los grupos sirven para reunir personas con intereses comunes, permitiendo, a diferencia de las páginas, la posibilidad de establecer foros de discusión. Las páginas se crean con fines específicos, no contienen foros de discusión y están encaminadas hacia marcas o personajes. Cfr. http://es. wikipedia.org/wiki/Facebook [Recuperado 02-05-2011]. La página de inicio de cada cuenta de Facebook dispone de cinco posibilidades: informar sobre nuestro estado y actualizarlo, añadir enlaces, fotos, videos, o preguntas. El estado sirve para informar a los amigos cómo estamos o qué hacemos durante un determinado período de tiempo, mostrado a través de la pregunta "¿qué estás haciendo ahora?", tal y como se explica en una de las páginas de ayuda de la web, disponible en: www.ayudaenlaweb.com/redes-sociales/actualizar-tu-estado [Recuperado 06-05-2011].
} 


\section{Resultados}

Un total de 46 diputados, de los 75 que componen el Parlamento de Galicia, disponen de Facebook (38 disponibles de forma pública y 8 privada), lo que representa el $61,3 \%$ de la Cámara, frente al $38,6 \%$ de representantes que no disponen de esta herramienta.

\begin{tabular}{ll}
\hline Total Parlamento & 75 \\
Total Parlamentarios & 46 \\
\hline Parlamentarios/-as con Facebook & 29 \\
\hline Parlamentarios/-as sin Facebook & 38 \\
\hline Parlamentarios/-as con Facebook Público & 8 \\
\hline Parlamentarios/-as con Facebook Privado & \\
\hline Número Total de amigos & 70.328 \\
\hline Número Total de páginas & 2.073 \\
\hline Parlamentarios/-as con Facebook del PP & 25 \\
\hline Parlamentarios/-as con Facebook del PSdeG & 12 \\
\hline
\end{tabular}

\footnotetext{
${ }^{11}$ Se han considerado como de carácter privado las cuentas definidas como personales o no políticas por sus propios creadores y aquellas que desestimaron hasta tres solicitudes consecutivas de amistad.
} 


\section{Fuente: Elaboración propia}

Por partidos, el PP es la formación que dispone, en términos absolutos, de mayor número de parlamentarios con Facebook (25 sobre un total de 38 representantes), seguido del PSdeG-PSOE (12 sobre 25) y Bloque Nacionalista Galego (BNG), formación en la que 9 de sus 12 diputados disponen de esta herramienta.

Sin embargo, el BNG es la formación con mayor penetración de Facebook, ya que, proporcionalmente, dispone de más representantes que utilizan esta herramienta (75\% de sus diputados), seguido del PP $(65,79 \%)$ y PSdeG-PSOE (48\%).

Total Parlamentarios con Facebook por Partido

$\begin{array}{llll}\text { Partido } & \text { Parlamentarios } & \text { Con Facebook } & \text { Uso por partido } \\ \text { PP } & 38 & 25 & 65,79 \%\end{array}$

$\begin{array}{lccc}\text { PSOE } & 25 & 12 & 48 \% \\ \text { BNG } & 12 & 9 & 75 \%\end{array}$

$\begin{array}{lll}\text { TOTAL } & 75 & 46\end{array}$

Fuente: Elaboración propia

REDMARKA UIMA-Universidad de A Coruña - CIECID 
En cuanto al número total de amigos por partido en Facebook, el Grupo Parlamentario Popular es el que acumula mayor número, con un total de 32.128 amigos agregados a sus 38 diputados, seguido del PSdeG (21.092) y BNG (17.108). A pesar de la Cámara gallega no dispone, como institución, de enlace de Facebook, el conjunto de los diputados de los tres partidos con representación en el hemiciclo logran sumar un total de 70.328 amigos a través de esta red social.

En cuanto al número de páginas que siguen los parlamentarios (a través del botón 'me gusta'), sobre un total de 2.073 páginas, los parlamentarios con Facebook muestran los siguientes números: 828 páginas los miembros del PP, 809 el PSdeG y 436 el BNG.

Por grupos parlamentarios y diputados, el ranking de los cinco primeros según el número de amigos agregados a sus cuentas de Facebook está encabezado por el presidente de la Xunta y diputado del PP Alberto Núñez Feijoo (9.361 amigos), seguido de la diputada socialista María del Mar Barcón Sánchez (5.003), que ocupa el segundo puesto, prácticamente igualada con el diputado del PP José Manuel Baltar Blanco (5.002). En cuarto lugar, se sitúa el que fuera vicepresidente de la Xunta en la anterior legislatura y diputado del BNG Anxo Quintana (4.564 amigos), seguido del diputado del PSdeG José Manuel Lage Tuñas, que constaba con un total de 4.370 amigos agregados durante el período de realización de este estudio.

Ranking según número de amigos

REDMARKA UIMA-Universidad de A Coruña - CIECID 


\begin{tabular}{llll}
\hline Posición & Parlamentario/-a & Partido & Amigos \\
1 & Nuñez Feijóo, Alberto ${ }^{12}$ & PP & 9.361 \\
2 & Barcón Sánchez, María del Mar & PSdeG-PSOE & 5.003 \\
3 & Baltar Blanco, José Manuel & PP & 5.002 \\
4 & Quintana González, Anxo Manuel & BNG & 4.564 \\
5 & Lage Tuñas, José Manuel & PSdeG-PSOE & 4.370 \\
\hline
\end{tabular}

Fuente: Elaboración propia

Si partimos de que el hemiciclo gallego dispone de un total de 75 escaños y teniendo en cuenta que son 46 los diputados gallegos que disponen de Facebook, podemos extrapolar cuál sería, en función de la presencia en esta red social, la representación que correspondería a los diputados de cada formación ${ }^{13}$, de tal modo que el PP subiría tres escaños (pasando de 38 a 41), los mismos que el BNG (de 12 a 15). En consecuencia, seis actas ganadas por populares y nacionalistas a costa de los socialistas, que pasarán de 25 a 19 escaños. La gráfica siguiente muestra el resultado de la extrapolación:

\section{Representatividad actual}

\section{Representatividad extrapolada}

\footnotetext{
${ }^{12}$ Cabe matizar que el presidente de la Xunta, Alberto Núñez Feijóo, dispone de dos perfiles en Facebook y de una página, lo que justifica en parte la acumulación de tal cantidad de amigos agregados a su cuenta.

${ }^{13}$ Una simple regla de tres nos permitiría despejar cuántos escaños, sobre el total de los 75 existentes, corresponderían a los 25 diputados del PP, los 12 del PSdeG y los 9 del BNG que disponen de Facebook.
} 


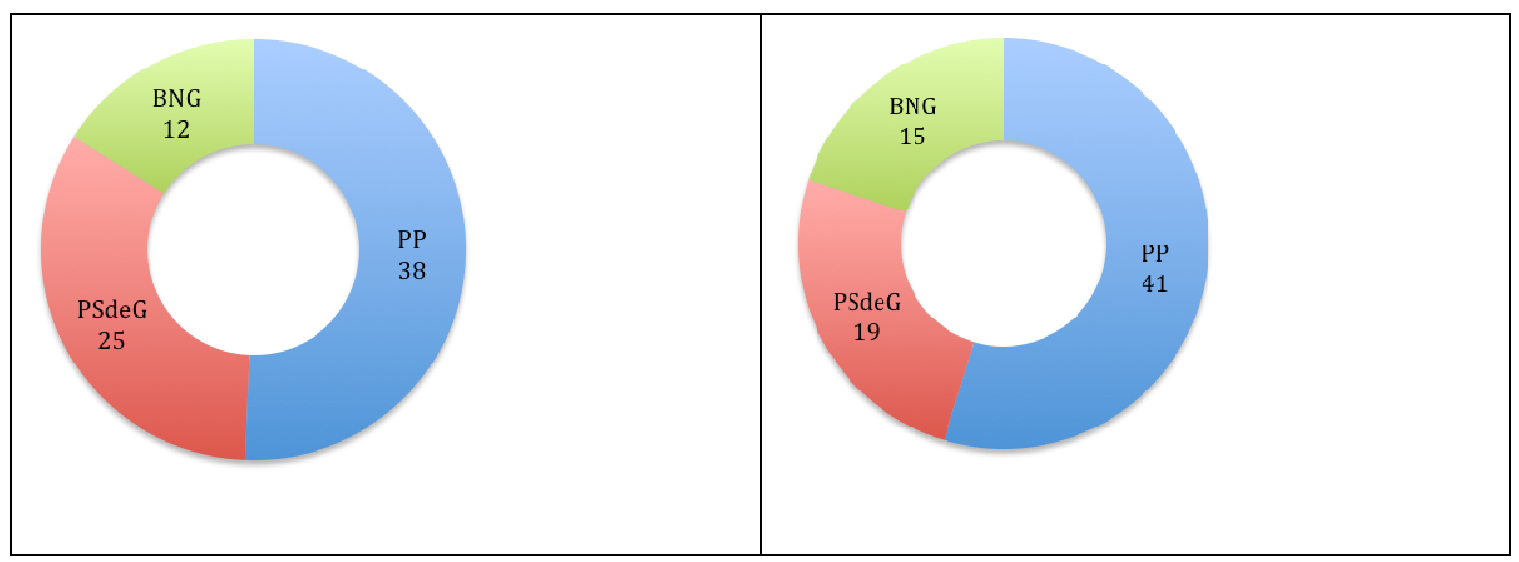

Fuente: Elaboración propia

Sin embargo, entre los parlamentarios con Facebook, los representantes del PSdeG-PSOE son los más "activos", ocupando los cinco primeros puestos, tal y como revela la media de todas sus actualizaciones (de estado, enlaces, fotografías y vídeos), durante los cinco meses analizados ${ }^{14}$.

\section{Gráfico de las actualizaciones medias}

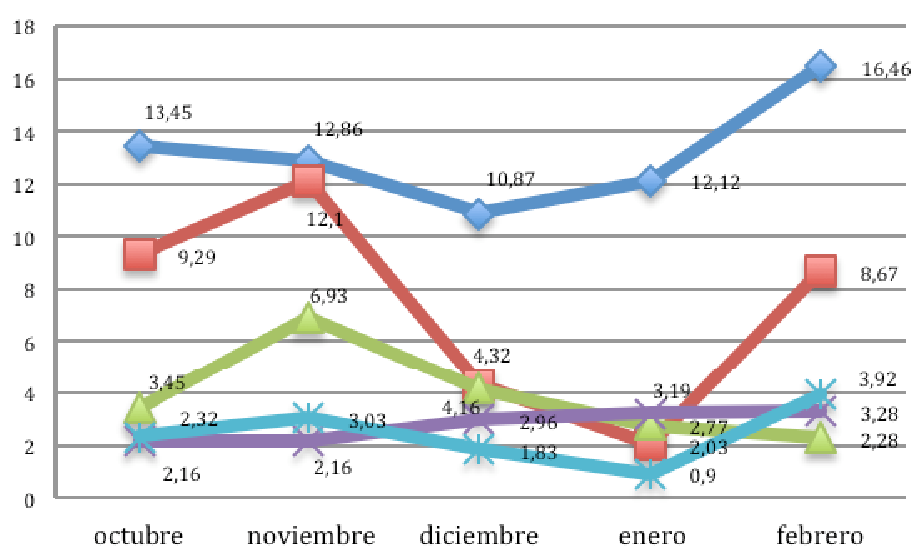

Barcón Sánchez, María del Mar

$\square$ Sestayo Doce, Beatriz

Lage Tuñas, José Manuel

Soneira Tajes, María Soledad

Fraga Santos, Silvia Belén

\section{Fuente: Elaboración propia}

${ }^{14}$ El cálculo de la media se obtuvo sobre el cómputo total diario de actualizaciones (la suma de las cuatro variables de estado, enlaces, fotografías y vídeo), dividido entre el período de estudio (151 días), obteniendo de este modo la frecuencia.

REDMARKA UIMA-Universidad de A Coruña - CIECID

Año IV, Número 7, (2011), pp. 77-106

http://www.redmarka.org/

$$
\text { ISSN 1852-2300 }
$$


A continuación puede verse la tabla con el ranking de todos los parlamentarios que realizaron actualizaciones de algún tipo, donde se puede observar que sólo el $28,2 \%$ de los diputados con Facebook (13 de 46), logran superar la media de una actualización por día. De ellos, 6 diputados son del PSdeG, 4 del PP y 3 del BNG. Además, más de la tercera parte de los parlamentarios con Facebook, muestran una presencia cuasi testimonial, por debajo del 0,1 de media diaria.

\begin{tabular}{llll}
\hline Ranking por actualización & & \\
Posición & Parlamentario/-a & Partido & $\begin{array}{l}\text { Actualizaciones } \\
\text { diarias }\end{array}$ \\
1 & Barcón Sánchez, María del Mar & PSdeG-PSOE & 13,09 \\
2 & Sestayo Doce, Beatriz & PSdeG-PSOE & 7,23 \\
3 & Lage Tuñas, José Manuel & PSdeG-PSOE & 3,93 \\
4 & Soneira Tajes, María Soledad & PSdeG-PSOE & 2,75 \\
5 & Fraga Santos, Silvia Belén & PSdeG-PSOE & 2,37 \\
6 & Baltar Blanco, José Manuel & PP & 2,32 \\
7 & Vázquez Fernández, Manuel & PSdeG-PSOE & 1,64 \\
8 & Adán Villamarín, Carme & BNG & 1,48 \\
9 & Álvarez Chao, Enma & PP & 1,43 \\
\hline & & &
\end{tabular}




\begin{tabular}{|c|c|c|c|}
\hline 10 & Negreira Souto, Carlos & $\mathrm{PP}$ & 1,41 \\
\hline 11 & Pontón Mondelo, Ana Belén & BNG & 1,24 \\
\hline 12 & Bouza Santiago, Ana Luisa & BNG & 1,22 \\
\hline 13 & Díaz Lugilde, Yolanda & PP & 1,05 \\
\hline 14 & Cajide Hervés, Carmen & PSdeG-PSOE & 0,9 \\
\hline 15 & Castiñeira Broz, Jaime & PP & 0,79 \\
\hline 16 & Aymerich Cano, Carlos & $\mathrm{BNG}$ & 0,7 \\
\hline 17 & $\begin{array}{l}\text { Fernández Fernández, Rosendo } \\
\text { Luís }\end{array}$ & PP & 0,56 \\
\hline 18 & Goldar Güimil, Jesús & PP & 0,56 \\
\hline 19 & Rodríguez Miranda, Antonio & PP & 0,48 \\
\hline 20 & Valcárcel Gómez, Marta & PP & 0,33 \\
\hline 21 & $\begin{array}{l}\text { Cobián Fernández de la Puente, } \\
\text { Pablo }\end{array}$ & PP & 0,31 \\
\hline 22 & Prado del Río, Paula & PP & 0,28 \\
\hline 23 & López Campos, José Carlos & PP & 0,17 \\
\hline 24 & Méndez Romeu, José Luis & PSdeG-PSOE & 0,17 \\
\hline 25 & Sueiro Pastoriza, Alberto & PP & 0,15 \\
\hline 26 & Verdes Gil, Sonia & PSdeG-PSOE & 0,14 \\
\hline 27 & Acuña do Campo, Carmen & PSdeG-PSOE & 0,13 \\
\hline
\end{tabular}




\begin{tabular}{|c|c|c|c|}
\hline 28 & Núñez Feijóo, Alberto & $\mathrm{PP}$ & 0,11 \\
\hline 29 & Escribano Rodríguez, Javier & PP & 0,09 \\
\hline 30 & Quintana González, Anxo Manuel & BNG & 0,07 \\
\hline 31 & Pose Mesura, Modesto & PSdeG-PSOE & 0,06 \\
\hline 32 & García López, José Antonio & PP & 0,05 \\
\hline 33 & Fernández Leiceaga, Xaquín & PSdeG-PSOE & 0,04 \\
\hline 34 & Fervenza Costas, José & PP & 0,03 \\
\hline 35 & Seoane Romero, María & PP & 0,03 \\
\hline 36 & Paz Franco, Tereixa & BNG & 0,03 \\
\hline 37 & Romero Fernández, Cristina & PP & 0,02 \\
\hline 38 & Táboas Veleiro, Teresa & BNG & 0,02 \\
\hline 39 & Rodríguez González, Román & PP & 0,01 \\
\hline 40 & Lobeira Domínguez, Bieito & BNG & 0 \\
\hline
\end{tabular}

Fuente: Elaboración propia

El propio presidente de la Xunta, Alberto Núñez Feijoo, presenta una media de 0,11 actualizaciones diarias durante el período analizado, ocupando el puesto número 28 , muy por detrás del diputado y secretario general de los socialistas 
gallegos, Manuel Vázquez, que ocupa el puesto número 7, y del portavoz parlamentario del BNG, Carlos Aymerich ${ }^{15}$, situado en el puesto 16.

\section{RESPONSABLES POLITICOS DE LOS TRES GRUPOS PARLAMENTARIOS}

\begin{tabular}{|c|c|c|c|c|c|c|}
\hline Nombre & Ranking & Variables & $\begin{array}{l}\text { Total por } \\
\text { variable }\end{array}$ & $\begin{array}{l}\text { Media } \\
\text { diaria por } \\
\text { variable }\end{array}$ & Total & $\begin{array}{l}\text { Media diaria de } \\
\text { actualizaciones }\end{array}$ \\
\hline \multirow{4}{*}{$\begin{array}{l}\text { Vázquez } \\
\text { Fernández } \\
\text {, Manuel }\end{array}$} & \multirow{4}{*}{7} & $\begin{array}{l}\text { Actualizaciones } \\
\text { de estado }\end{array}$ & 115 & 0,76 & \multirow{4}{*}{248} & \multirow{4}{*}{1,64} \\
\hline & & Enlaces & 23 & 0,15 & & \\
\hline & & Fotografías & 94 & 0,62 & & \\
\hline & & Video & 16 & 0,11 & & \\
\hline \multirow{5}{*}{$\begin{array}{l}\text { Aymerich } \\
\text { Cano, } \\
\text { Carlos }\end{array}$} & \multirow{4}{*}{16} & $\begin{array}{l}\text { Actualizaciones } \\
\text { de estado }\end{array}$ & 34 & 0,23 & \multirow{4}{*}{106} & \multirow{4}{*}{0,70} \\
\hline & & Enlaces & 38 & 0,25 & & \\
\hline & & Fotografías & 32 & 0,21 & & \\
\hline & & Video & 2 & 0,01 & & \\
\hline & & $\begin{array}{l}\text { Actualizaciones } \\
\text { de estado }\end{array}$ & 12 & 0,08 & & \\
\hline
\end{tabular}

${ }^{15}$ Se contabiliza el Parlamentario Carlos Aymerich, en calidad de Portavoz del Grupo Parlamentario del BNG, pues el Portavoz Nacional de esta formación, Guillerme Vázquez, carece de escaño en la Cámara.

REDMARKA UIMA-Universidad de A Coruña - CIECID 


\begin{tabular}{|c|c|c|c|c|c|c|}
\hline \multirow{3}{*}{$\begin{array}{l}\text { Núñez } \\
\text { Feijóo, } \\
\text { Alberto }\end{array}$} & & Enlaces & 0 & 0,00 & \multirow{3}{*}{16} & \multirow{3}{*}{0,11} \\
\hline & & Fotografías & 3 & 0,02 & & \\
\hline & 28 & Video & 1 & 0,01 & & \\
\hline
\end{tabular}

Fuente: Elaboración propia

Desgranando cada una de las cuatro variables consideradas, obtenemos los siguientes rankings de los cinco parlamentarios situados en los primeros puestos en cuanto a actualización de estados, enlaces, fotografías y vídeos ${ }^{16}$.

\section{Ranking por actualización de estados}

\begin{tabular}{llll} 
Posición & Parlamentario/-a & Partido & $\begin{array}{l}\text { Actualizaciones } \\
\text { de }\end{array}$ \\
& & estado diarias \\
1 & Barcón Sánchez, María del Mar & PSdeG-PSOE & 6,39 \\
3 & Sestayo Doce, Beatriz & PSdeG-PSOE & 4,52 \\
\hline 3 & Lage Tuñas, José Manuel & PSdeG-PSOE & 2,18
\end{tabular}

${ }^{16}$ Existen una serie de herramientas y "trucos" que facilitan la actualización automática de estado, sin necesidad de introducir ningún comentario. Servicios que permiten programar actualizaciones de fecha y hora como "Later Bro" o "Facebook Places" y "Foursquare", sistemas de geolocalización a través del teléfono móvil. A la hora de realizar este trabajo estas herramientas se intentaron excluir, en la medida de lo posible, del cómputo, del mismo modo que los enlaces, fotografías y vídeos subidos por terceras personas en la cuenta de cada parlamentario. Tampoco se contabilizaron la multitud de aplicaciones y juegos disponibles. 


\begin{tabular}{lllc}
\hline 4 & Fraga Santos, Silvia Belén & PSdeG-PSOE & 1,17 \\
5 & Baltar Blanco, José Manuel & PP & 0,91 \\
\hline
\end{tabular}

Fuente: Elaboración propia

2.- Enlaces:

\section{Ranking de publicación de enlaces diarios}

\begin{tabular}{llll} 
Posición & Parlamentario/-a & Partido & $\begin{array}{l}\text { Actualizaciones de } \\
\text { enlaces diarias }\end{array}$ \\
\hline 1 & Barcón Sánchez, María del Mar & PSdeG-PSOE & 6,15 \\
\hline 2 & Soneira Tajes, María Soledad & PSdeG-PSOE & 2,45 \\
\hline 3 & Lage Tuñas, José Manuel & PSdeG-PSOE & 1,48 \\
\hline 4 & Adán Villamarín, Carme & BNG & 1,11 \\
& & & 0,93
\end{tabular}

Fuente: Elaboración propia

3.- Fotografías

\section{Ranking por publicaciones de fotografías diarias}

\begin{tabular}{llll} 
Posición & Parlamentario/-a & Partido & $\begin{array}{l}\text { Actualizaciones de } \\
\text { fotografías diarias }\end{array}$ \\
\hline 1 & Sestayo Doce, Beatriz & PSdeG-PSOE & 1,42 \\
\hline 2 & Baltar Blanco, José Manuel & PP & 0,77 \\
\hline
\end{tabular}




\begin{tabular}{llll}
\hline 3 & Negreira Souto, Carlos & PP & 0,73 \\
4 & Vázquez Fernández, Manuel & PSdeG-PSOE & 0,62 \\
5 & Castiñeira Broz, Jaime & PP & 0,31 \\
\hline
\end{tabular}

Fuente: Elaboración propia

4.- Videos:

\section{Ranking por publicaciones de video diaria}

\begin{tabular}{|llll}
\hline Posición & Parlamentario/-a & Partido & Videos diarios \\
\hline 1 & Sestayo Doce, Beatriz & PSdeG-PSOE & 0,55 \\
2 & Baltar Blanco, José Manuel & PP & 0,43 \\
3 & Díaz Lugilde, Yolanda & PP & 0,42
\end{tabular}

\begin{tabular}{llll}
4 & Barcón Sánchez, María del Mar & PSdeG-PSOE & 0,37 \\
5 & Álvarez Chao, Enma & PP & 0,32 \\
\hline
\end{tabular}

Fuente: Elaboración propia

En definitiva, en el caso del Parlamento de Galicia, el perfil del diputado medio es el de un político con una media de 1.647 amigos agregados a su cuenta de Facebook, que sigue casi medio centenar de páginas y con una constancia de 1,21 actualizaciones diarias. 


\section{Perfil medio del parlamentario/-a}

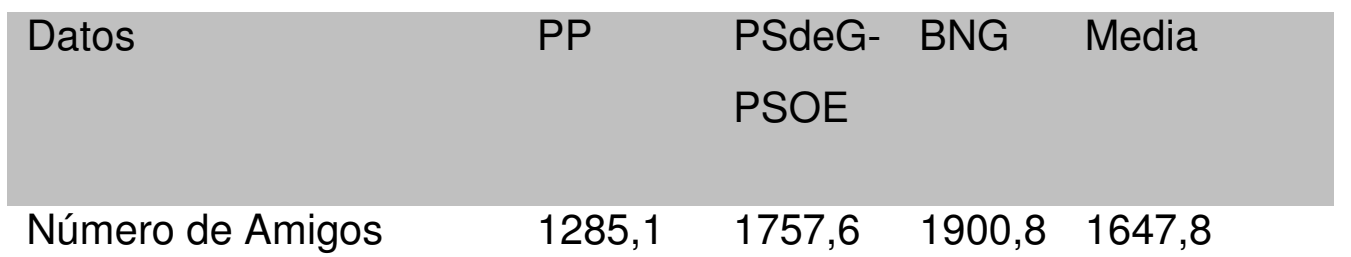

\begin{tabular}{lllll} 
Número de Páginas & 33,12 & 67,41 & 48,44 & 49,6 \\
\hline Actualizaciones diarias & 0,4 & 2,7 & 0,53 & $1,21^{17}$
\end{tabular}

Fuente: Elaboración propia

En cuanto a la presencia en Facebook por sexos, nos encontramos con que el número de parlamentarios gallegos que utilizan esta herramienta supera, ligeramente, al de las diputadas (54\% de hombres frente al $46 \%$ de las mujeres), una proporción descompensada, sobre todo, por la diferencia existente en el caso de Grupo Parlamentario Popular (16 hombres con Facebook frente a 9 mujeres), mientras que en el PSdeG y BNG, el número de diputadas que emplean esta herramienta supera, ligeramente, a la de diputados, tal y como se puede comprobar en la tabla siguiente.

Uso de Facebook por partido y sexo

\begin{tabular}{|c|c|c|c|c|c|}
\hline \multirow{3}{*}{ Partido } & \multirow{3}{*}{ Número de Parlamentarios } & \multicolumn{2}{|c|}{ Parlamentarios } & \multicolumn{2}{|c|}{ Parlamentarias } \\
\hline & & Total & Con & Total & Con \\
\hline & & & Facebook & & Facebook \\
\hline
\end{tabular}

${ }^{17}$ Si la media se eleva en este caso es gracias al "top" de los cinco diputados más activos del Parlamento gallegos, encabezados por la parlamentaria socialista Mar Barcón que, como se refleja en la gráfica y tabla mostrada con anterioridad, supera las dos actualizaciones por hora al día. 


\begin{tabular}{lcccccc}
\hline PP & 38 & 25 & 16 & 13 & 9 \\
PSOE & 25 & 14 & 5 & 11 & 7 \\
BNG & 12 & 6 & 4 & 6 & 5 \\
TOTAL & 75 & 45 & 25 & 30 & 21 \\
\hline
\end{tabular}

Fuente: Elaboración propia

Por lo que respecta al idioma, el porcentaje de los diputados con Facebook que emplean únicamente el español es del $60 \%$ en el caso del PP y del $25 \%$ en el PSdeG-PSOE, mientras que en el BNG todos sus representantes emplean, únicamente, el gallego.

\section{Idioma y número de parlamentarios/-as}

$\begin{array}{llll} & \text { Bilingüe } & \text { Español } & \text { Gallego } \\ \text { PP } & 8 & 15 & 2\end{array}$

$\begin{array}{llll}\text { PSdeG-PSOE } & 4 & 3 & 5 \\ \text { BNG } & & 9\end{array}$

\begin{tabular}{|c|c|c|c|}
\hline Total & 12 & 18 & 16 \\
\hline
\end{tabular}

Finalmente, y aunque se trate de un estudio básicamente cuantitativo, se observaron los grupos agregados por los distintos parlamentarios y sus 
comentarios al respecto. Teniendo en cuenta el período de estudio, se encontraron algunas coincidencias entre los diputados de los tres grupos con representación en el hemiciclo gallego.

Así, en el caso del Partido Popular, varios son los que mostraron su apoyo a la iglesia católica y la visita del Papa Benedicto XVI a Galicia (Pablo Cobián, Jaime Castiñeira, Yolanda Díaz, Antonio Rodríguez, Paula Prado), y a favor de las plataformas en contra del paro, reclamando la dimisión del presidente del Gobierno, José Luis Rodríguez Zapatero, y la convocatoria de elecciones generales anticipadas (Emma Álvarez, Jaime Castiñeira, Yolanda Díaz, María Ángeles Díaz, Rosendo Luis Fernández, Jesús Goldar, Paula Prado y María Seoane).

Grandes apoyos recibe también el grupo de quienes se pronuncian en el PP en contra de la subida del IVA (Emma Álvarez, Jaime Castiñeira, María Ángeles Díaz, Jesús Goldar, Paula Prado y María Seoane), mientras que dos parlamentarios de esta formación muestran su apoyo al topónimo español de la ciudad de La Coruña, en lugar del gallego A Coruña (Pablo Cobián y Carlos Negreira) y otros dos proponen el cambio de las sedes del sindicato UGT por comida para África (Yolanda Díaz y Marisol Piñeiro).

Por lo que respecta al PSdeG-PSOE, las mayores coincidencias se registraron en el grupo de apoyo al juez Baltasar Garzón (Mar Barcón, Carmen Cajide, Modesto Pose y Marisol Soneira) y el colectivo "Arde Galicia", de defensa del monte gallego frente a los incendios (José Manuel Lage, Sonia Verdes).

En el BNG destaca el grupo que reivindica la enseñanza en gallego y las plataformas de apoyo del idioma gallego, que respaldan 5 de los 9 diputados de esta formación que disponen de Facebook (Carme Adán, Tereixa Paz, Ana Belén Pontón, Anxo Quintana y Henrique Viéitez), teniendo en cuenta además que otros dos representantes de la formación nacionalista exigen la renuncia de la diputada de UPyD, Rosa Díez, por considerarla racista (Carme Adán y Ana Luisa Bouza). Dos parlamentarios del BNG apoyan igualmente la plataforma en favor de la selección gallega de fútbol (Ana Luisa Bouza y Ana Belén Pontón), y el portavoz 
de esta formación, Carlos Aymerich, la plataforma de defensa de la sanidad pública.

\section{Conclusiones}

1. Las encuestas y datos de audiencias muestran, en comparación con los estudios sobre el empleo de las redes sociales por parte de los parlamentarios, que el ritmo de evolución en la utilización de estas herramientas no es el mismo, mostrando más crecimiento en el ámbito social que político.

2. Los datos de los diputados gallegos que disponen de Facebook muestran una presencia mayor que la de sus homólogos en el Congreso de los Diputados. Más de dos terceras partes de los representantes gallegos disponen de esta herramienta, frente a la escasa tercera parte de los parlamentarios estatales que referencian alguna actividad en las redes sociales y la cuarta parte $(25,14 \%)$ de los que disponen de perfil o página personal en el caso concreto de Facebook.

3. Sin embargo, las distancias se acortan al comprobar la actividad y utilización de Facebook por parte de los diputados gallegos con respecto a los miembros del Congreso de los Diputados, pues apenas la tercera parte de los gallegos alcanzaron una media de una actualización diaria durante el período analizado y, en el caso de los restantes, su utilización es prácticamente testimonial, al igual que ocurre con los representantes estatales.

4. Por grupos parlamentarios, los representantes del Partido Popular son, en términos absolutos, los que muestran mayor presencia en Facebook, si bien, proporcionalmente, el BNG es la formación con mayor presencia en esta herramienta. De hecho, si extrapolásemos el reparto de los 75 escaños del hemiciclo gallego en función de los parlamentarios que disponen Facebook en cada partido, el resultado sería que PP y BNG ganarían tres escaños cada uno, a costa de los seis que perdería el PSdeG-PSOE. Sin embargo, también nos 
encontramos con que los cinco diputados más activos en la utilización de Facebook son todos del Grupo Socialista, lo cual muestra la gran descompensación existente en el PSdeG-PSOE en la utilización de esta herramienta. Mayor presencia, por tanto, en el caso de los representantes gallegos con respecto a los parlamentarios del Congreso, pero similar y escasa utilización de esta herramienta por parte de ambos, tal y como muestran sus respectivas medias de actualizaciones.

5. Los diputados del Congreso fueron incorporándose, progresivamente, a la utilización de páginas webs y blogs, pero los representantes gallegos lo hicieron en mayor medida a las nuevas herramientas de las redes sociales, al menos por lo que respecta al caso de Facebook.

\section{BIBLIOGRAFÍA}

Anduiza, Eva, et al. (2010). "Los usos políticos de Internet en España”. Revista Española de Investigaciones Sociológicas (Reis), no129, pp. 133-146.

Castells, Manuel (2009). Comunicación y poder. Madrid: Alianza Editorial.

Castells, Manuel (2001). La Galaxia Internet. Reflexiones sobre Internet, empresa y sociedad. Barcelona: Areté.

Congosto, M. Luz et al. (2011). "Twitter y política: Información, opinión y ¿predicción?”. Cuadernos de Comunicación Evoca. Comunicación política 2.0., n-4, pp. 11-15.

Cotarelo, Ramón (2010). La política en la era de Internet. Valencia: Tirant lo Blanch.

Dader, José Luis y Campos, Eva (2006). “Internet parlamentario en España (1999-2005): Los recursos para el contacto ciudadano y su uso, con una comparación europea”. ZER, Revista de Estudios de comunicación, №18, p.105.

Dader, José Luis (2002). "La ciberdemocracia posible: reflexión prospectiva a partir de la experiencia en España". Cuadernos de Información y Comunicación (CIC), n6, pp.177- 220. 
Dahlgren, P. (2005). "The Internet, Public Spheres and Political Communication: Dispersion and Deliberation". Political Communication, №22, pp.147-162.

Drezner, Daniel y Farrell, Henry (2008). "Introduction: Blogs, politics and power: a special issue of Public Choice". Public Choice, no 134, pp. 1-13.

Fages-Ramió, R. "Actitud 2.0: la política más allá de los blogs". Revista de Internet, Derecho y Política, №7, pp. 19-25.

Fernández Crespo, M. (2007). Internet, Blogs y Política 2.0. Madrid: Fundación Jaime Vera.

Larrañaga Rubio, J. (2008). "Análisis económico de la evolución de las audiencias de los medios tradicionales e impacto de Internet en su consumo". Área Abierta, n22, pp.1-18.

Resina de la Fuente, J. (2010). "Ciberpolítica, redes sociales y nuevas movilizaciones en España: el impacto digital en los procesos de deliberación y participación ciudadana". Mediciones sociales. Revista de Ciencias Sociales y de la Comunicación, ㄲo7, pp.144-164.

Túñez, M. y Sixto, J. (2011). "Redes sociales, política y Compromiso 2.0: La comunicación de los diputados españoles en Facebook". Revista Latina de Comunicación Social, ํo66, pp.210-234.

Rúas, J. (2011). Manual del Candidato Electoral. Madrid: Los Libros de la Catarata.

Rubio, R. et al. (2011). "Nuevas tecnologías y transparencia parlamentaria". Cuadernos de Comunicación Evoca. Comunicación política 2.0., n4, pp. 11-15.

Para citar este artículo:

Rúas Araújo, José - Dapena González, Borja (15-12-2011). LOS DIPUTADOS DEL

PARLAMENTO GALLEGO EN FACEBOOK.

REDMARKA - CIECID - Unidad de Investigación en Marketing Aplicado-Universidad de A Coruña

Año IV, Número 7, V1, pp.77-106

ISSN $1852-2300$

URL del Documento: cienciared.com.ar/ra/doc.php? $\mathrm{n}=1597$

URL de la Revista: cienciared.com.ar/ra/revista.php?wid=39 